\title{
Fibroelastoma en cuerdas tendinosas de la válvula mitral como causa de ictus embólico
}

\author{
Iñigo Pereiro Lili \\ Nora García Ibarrondo \\ Arantza Manzanal Rey \\ Mireia Codina Prat \\ Lara Ruiz Gómez
}

Correspondencia

Iñigo Pereiro Lili

inigopereiro@hotmail.com

Servicio de Cardiología. Hospital Universitario de Basurto. Bilbao. País Vasco, España

Recibido: 15/12/2020

Aceptado:24/01/2021

En línea: 30/04/2021

Citar como: Pereiro Lili I, García Ibarrondo N, Manzanal Rey A, Codina Prat M, Ruiz Gómez L. Fibroelastoma en cuerdas tendinosas de la válvula mitral como causa de ictus embólico. Rev Ecocar Pract (RETIC). 2021 (Abril); 4 (1): 26-29. doi: 10.37615/retic.v4n1a10.

Cite this as: Lili I, García Ibarrondo N, Manzanal Rey A, Codina Prat M, Ruiz Gómez L. Fibroelastoma in the chordae tendineae of the mitral valve as a cause of embolic stroke. Rev Ecocar Pract (RETIC). 2021 (Abril); 4 (1): 26-29. doi: 10.37615/retic.v4n1a10.

\section{Palabras clave}

$\triangleright$ Fibroelastoma mitral

$\triangleright$ Ictus

\section{RESUMEN}

Presentamos 2 casos de fibroelastoma cardíaco localizado sobre las cuerdas tendinosas de la válvula mitral que debutaron como ictus embólico. En ambos casos, el diagnóstico de presunción fue ecocardiográfico, se intervinieron quirúrgicamente quirúrgica y se confirmó la sospecha inicial en el estudio histológico. El fibroelastoma es el segundo tumor cardíaco primario más frecuente y una causa conocida de ictus y embolias. Sin embargo, el manejo clínico es muchas veces controvertido debido a que en realidad son tumores infrecuentes y hay poca evidencia sobre las opciones de tratamiento.

\section{Primer caso}

Varón de 66 años con antecedentes de tabaquismo y consumo de alcohol ocasional sin factores de riesgo cardiovascular conocidos. Como único antecedente relevante tenía un trastorno depresivo recurrente en seguimiento por psiquiatría tratado con fluvoxamina y lorazepam.

Acude al servicio de Urgencias por un cuadro de más de 24 horas de evolución de mareo, torpeza leve en extremidades izquierdas e inestabilidad para la marcha.

Se realiza en urgencias un TAC de cráneo que muestra una lesión hipodensa cortico-subcortical en la región parasagital del lóbulo occipital derecho compatible con una lesión isquémica de reciente instauración junto a un pequeño defecto de repleción en la arteria cerebral posterior derecha compatible con trombo.
Dado que en el estudio de neuroimagen se apreciaron datos sugestivos de infarto establecido y que se había sobrepasado el tiempo de ventana terapéutica se desestimó el tratamiento agudo del ictus.

Ya en planta de hospitalización, se realizaron un estudio de troncos supra-aórticos y un Holter ECG sin resultados relevantes. Sin embargo, en el ecocardiograma trasntorácico (Video 1) se apreció una imagen móvil en las cuerdas tendinosas de la válvula mitral de unos 8-9 mm que podría corresponder con un fibroelastoma. Se realizó un ecocardiograma transesofágico (Video 2, Figura 1 y Video 3) que confirmó la sospecha inicial y no detectó otras posibles causas de embolia. Tras este hallazgo se decide realizar una resección quirúrgica de la lesión como probable desencadenante del cuadro del paciente. Fue intervenido 3 meses después con resección completa de la masa y confirmación histopatológica del diagnóstico de fibroelastoma. La evolución posterior fue favorable y no tuvo nuevos eventos embólicos.

La asociación de anomalías (válvula mitral en paracaídas, anillo supramitral y coartación aórtica) sugieren que se trataba de un complejo de Shone. 


\section{Estudio por imagen}

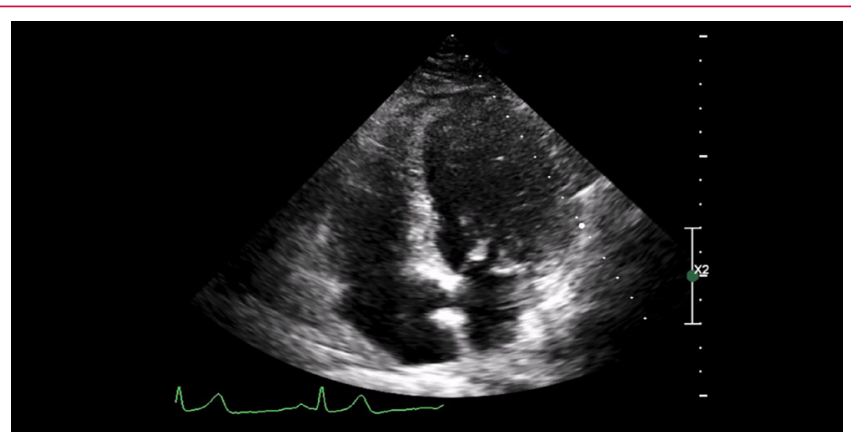

Vídeo 1. ETT plano apical de 4 cámaras. Se aprecia una imagen muy móvil en el aparato subvalvular mitral compatible con fibroelastoma

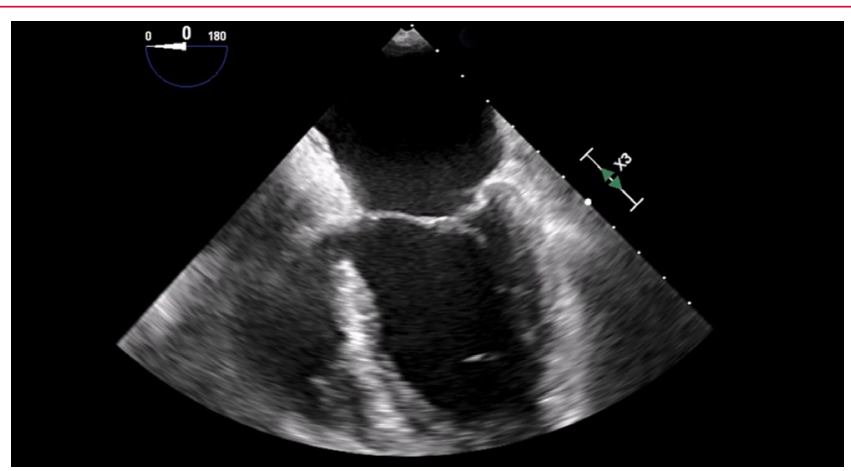

Vídeo 2. Ecocardiograma transesofágico. Plano transversal medio. Se aprecia con más detalle la imagen extraña apreciada en el estudio transtorácico en el aparato subvalvular mitral

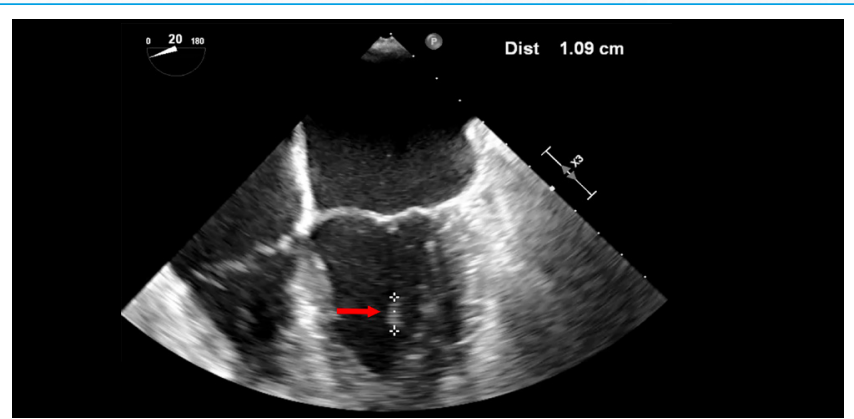

Figura 1. Ecocardiograma transesofágico. Se mide el diámetro mayor de la masa en $1,09 \mathrm{~cm}$.

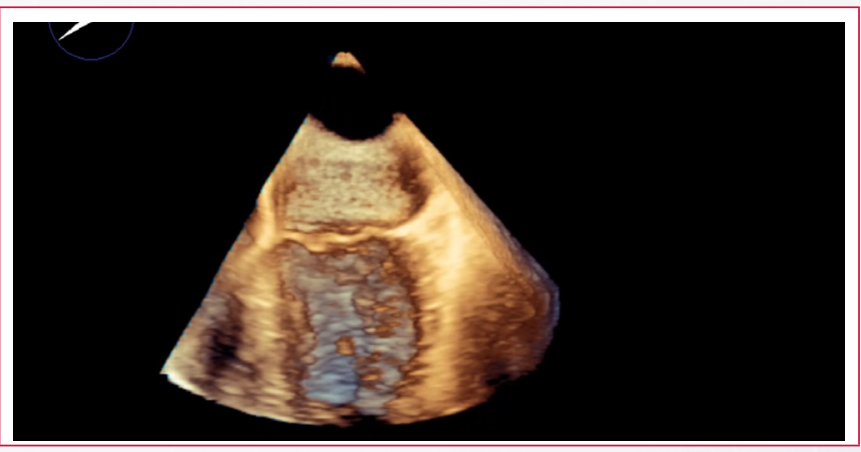

Vídeo 3. Estudio con 3D “Live-3D” de la lesión subvalvular mitral

\section{Segundo caso}

Mujer de 70 años sin antecedentes personales previos destacables. Acude al servicio de Urgencias por un cuadro de incapacidad de emisión del lenguaje sin déficit de comprensión ni otra focalidad neurológica acompañante, que cede de forma progresiva en 10-15 minutos.

Se realiza un TAC de cráneo que no mostró signos de patología intracraneal aguda. En la planta de hospitalización se solicita un estudio de troncos supraaórticos que no apreció alteraciones. Cuando se realizó el ecocardiograma transtorácico y transesofágico (Video 4, Video 5, video 6 y Figura 2) se detectó que la paciente tenía un flúter auricular con respuesta ventricular 4:1, con un ventrículo izquierdo no dilatado ni hipertrófico con FEVI global en el límite bajo (50\%). La válvula mitral presentaba alteraciones degenerativas con esclerosis de los velos y con regurgitación leve-moderada central. En el aparato subvalvular mitral, adherida a las cuerdas tendinosas, existía una pequeña imagen redondeada hiperecogénica e hipermóvil compatible con fibroelastoma.

Tras el hallazgo del flúter auricular se inició anticoagulación oral con acenocumarol y fue dada de alta hospitalaria con el diagnóstico neurológico de accidente isquémico transitorio. En los meses siguientes fue intervenida en otro centro hospitalario confirmando la anatomía patológica el fibroelastoma. Desde entonces sin nuevos eventos embólicos.

\section{Estudio por imagen}

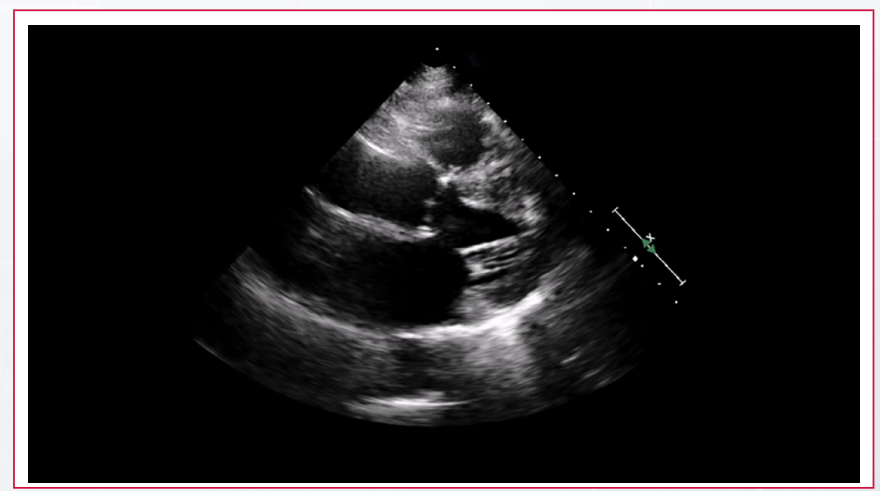

Vídeo 4. Estudio transtorácico en plano paraesternal eje largo. Se aprecia una masa vibrátil en aparato subvalvular mitral

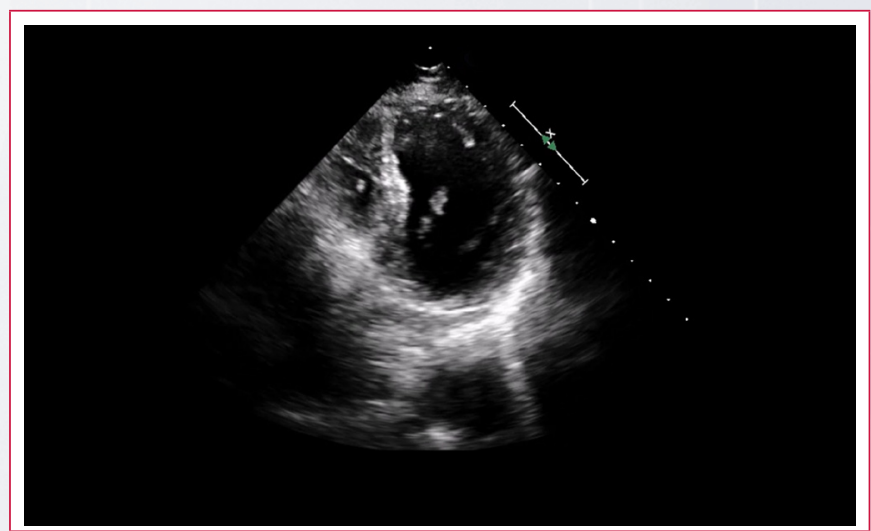

Vídeo 5. Plano apical de cámaras modificado. Nueva vista de la masa del aparato subvalvular mitral 


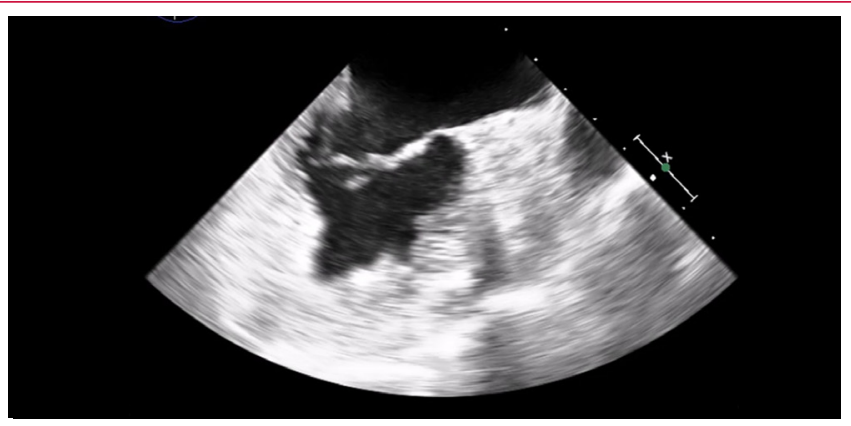

Vídeo 6. Ecocardiograma transesofágico. Plano longitudinal medio que permite valorar con más precisión la masa vista en el ecocardiograma transtorácico

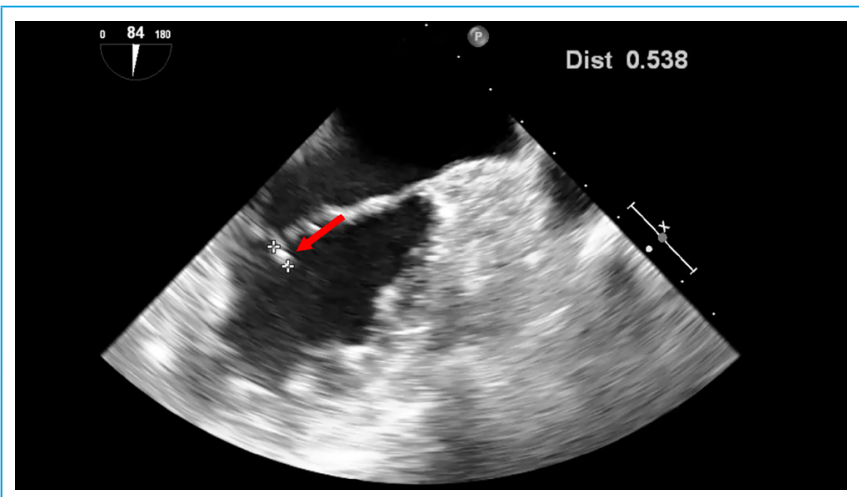

Figura 2. Ecocardiograma transesofágico. Se mide el diámetro mayor de la masa en tumoración es de $0.538 \mathrm{~cm}$

\section{Discusión}

El fibroelastoma cardíaco es un tumor cardíaco infrecuente. Existen trabajos que lo consideran el segundo tumor primario cardíaco más frecuente en adultos por detrás del mixoma, pero en realidad su prevalencia no es bien conocida (1). Los tumores varían en tamaño, desde 2 hasta $70 \mathrm{~mm}$ con una media de $9 \mathrm{~mm}$. El $80 \%$ de los fibroelastomas se localiza en las válvulas cardíacas, principalmente en las izquierdas (36\% válvula aórtica, $29 \%$ válvula mitral, $11 \%$ válvula tricúspide y $7 \%$ en válvula pulmonar). Cuando el tumor asienta sobre una válvula auriculo-ventricular suele tener predilección por localizarse en la cara auricular de las valvas ${ }^{(2)}$. La localización en las cuerdas tendinosas de la válvula mitral es muy rara, aunque existen varios casos descritos en la literatura3. La resección de estos tumores puede ocasionar una regurgitación mitral significativa, teniendo que proceder a posteriori a realizar una reparación/sustitución de valvular mitra|(3).

Este tipo de tumores se manifiestan con síntomas derivados de embolias, bien por rotura del propio tumor o por émbolos de fragmentos de fibrina y plaquetas que se pueden formar sobre los mismos ${ }^{(2)}$. La principal presentación clínica es el ictus o accidente isquémico transitorio, seguido por angina o infarto agudo de miocardio o muerte súbita, entre otros eventos.

El diagnóstico de presunción inicial es ecocardiográfico. Los fibroelastomas aparecen como una masa redondeada, con un pedículo estrecho y una zona central de mayor densidad ${ }^{(4)}$. Se debe hacer el diagnóstico diferencial con trombos, vegetaciones o excrecencias valvulares de Lamb|(4). En el caso de que el fibrolelastoma se origine en localizaciones atípicas el diagnóstico diferencial debe ampliarse para incluir otro tipo de masas. Por ejemplo, en este caso de fibroelastoma localizado en las cuerdas tendinosas el diagnóstico diferencial también debe incluir las cuerdas tendinosas aberrantes. En muchas ocasiones es necesario definir mejor la tumoración mediante ecocardiografía transesofágica ${ }^{(4)}$. El diagnóstico definitivo es histopatológico.

Con respecto al tratamiento no hay un consenso generalizado sobre qué hacer con estos pacientes. Klarich et al. en una serie publicada en 1997 no apreciaron un aumento de la mortalidad en pacientes asintomáticos tratados de forma conservadora ${ }^{(5)}$. Sin embargo, en una serie contemporánea publicada en 2015 por Tamin et al ${ }^{(1)}$ con 511 pacientes se comprobó que los pacientes con sospecha ecocardiográfica de fibroelastoma tenían un aumento del riesgo de ictus y de la mortalidad. Grinda et al y Ngaage et al. defienden realizar una escisión quirúrgica a todos los pacientes, estén o

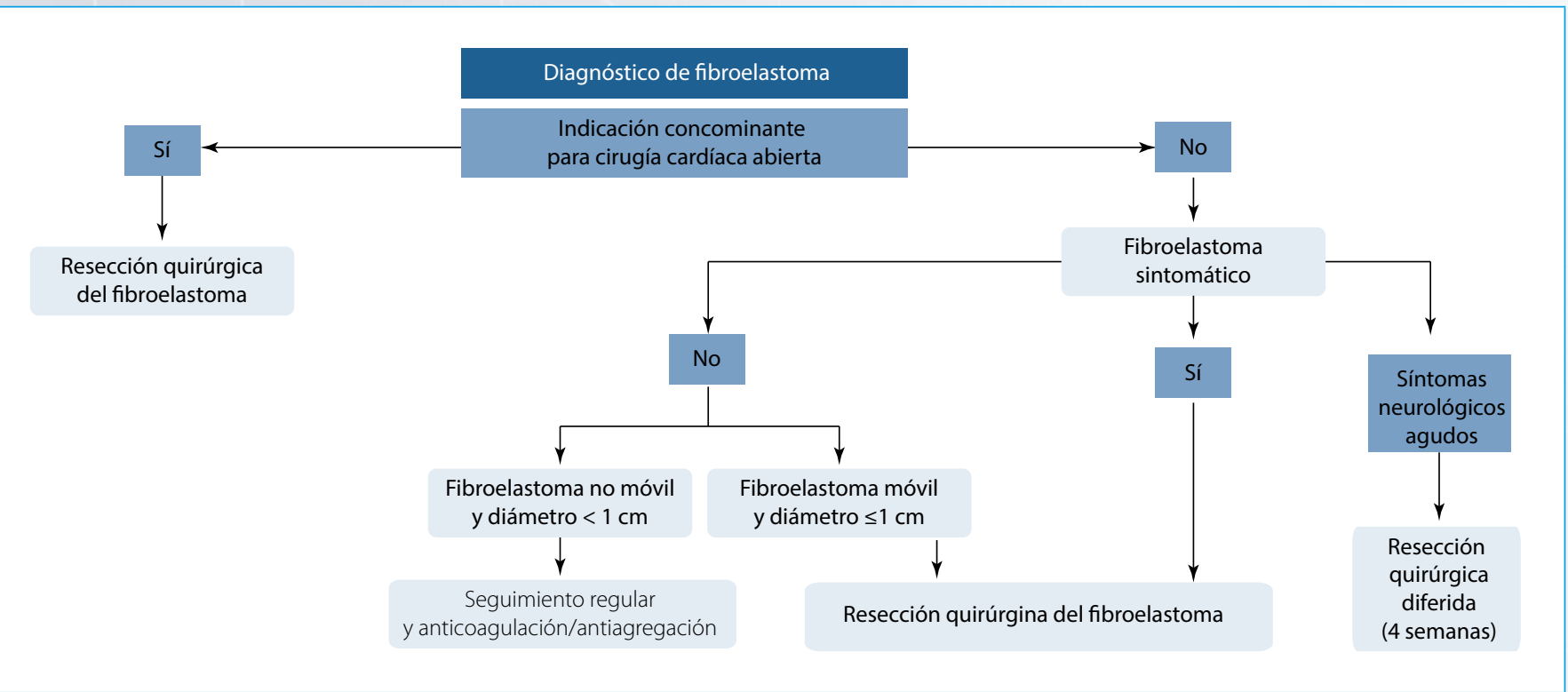

Figura 3. Algoritmo terapéutico adaptado de Mariscalco G, Bruno VD, Borsani P, Dominici C, Sala A. Papillary Fibroelastoma: Insight to a Primary Cardiac Valve Tumor. Journal of Cardiac Surgery. Marzo de 2010;25(2):198-205. 
no sintomáticos ${ }^{(6,7)}$. Existe un algoritmo de hace ya varios años (Figura 3 ) donde varios autores proponen una metodología de tratamiento(2).

En general se recomienda la cirugía de escisión para aquellos pacientes sintomáticos y también para aquellos que se van a someter a una cirugía cardíaca por otra razón. El gran debate sigue residiendo en los pacientes asintomáticos. Con respecto a los fibroelastomas de cavidades derechas, si no son sintomáticos hay autores que apuestan por no intervenirlos dado que raramente van a dar clínica ${ }^{(8)}$. En realidad, no se sabe cuál sería el manejo óptimo de estos tumores ya que no es posible realizar ensayos clínicos que comparen estrategias porque son tumores infrecuentes, así que no hay más evidencia que las recomendaciones de grupos e instituciones expertas como la Mayo Clinic.

\section{Conclusión}

El fibroelastoma cardíaco es el segundo tumor cardíaco primario más frecuente y una fuente ocasional de embolias sistémicas. La localización en las cuerdas tendinosas de la válvula mitral es rara.

El diagnóstico es inicialmente ecocardiográfico en la mayoría de los casos y el manejo es controvertido, sobre todo en aquellos pacientes asintomáticos. En pacientes con síntomas por embolismo muchas veces se opta por la cirugía de resección para disminuir el riesgo de nuevas embolias.

\section{Ideas para recordar}

- El fibroelastoma cardíaco es el segundo tumor primario cardíaco más frecuente.

- Es causa de eventos embólicos, siendo un origen conocido de ictus u otras embolias.

- El diagnóstico inicial es generalmente ecocardiográfico en la mayoría de los casos.
- El tratamiento es controvertido, pero en general, en aquellos pacientes sintomáticos o a los que van a ser intervenidos a cirugía cardíaca por otra razón se recomienda su extirpación.

\section{Bibliografía}

1. Tamin, S. S., Maleszewski, J. J., Scott, C. G., Khan, S. K., Edwards, W. D., Bruce, C.J.,Klarich, K. W. (2015). Prognostic and Bioepidemiologic Implications of Papillary Fibroelastomas. Journal of the American College of Cardiology, 65(22), 2420-2429. doi:10.1016/j.jacc.2015.03.569

2. Mariscal co G, Bruno VD, Borsani P, Dominici C, Sala A. Papillary Fibroelastoma: Insight to a Primary Cardiac Valve Tumor. Journal of Cardiac Surgery. Marzo de 2010;25(2):198-205.

3. Ziabakhsh S, Jalalian R, Mokhtari-Esbuie F. Papillary fibroelastoma of a mitral valve chordae, presenting with atypical chest pain and palpitation: A case report and the literature. Caspian J Intern Med. 2014;5(2):123-6.

4. Evangelista-Masip A. Manual de ecocardiografía clínica. España: CTO EDITORIAL; 2018.

5. Klarich KW, Enriquez-Sarano M, Gura GM, Edwards WD, Tajik AJ, Seward JB. Papillary Fibroelastoma: Echocardiographic Characteristics for Diagnosis and Pathologic Correlation. Journal of the American College of Cardiology. 1997;30(3):784-90

6. Grinda J-M, Couetil JP, Chauvaud S, D'Attellis N, Berrebi A, Fabiani J-N, et al. Cardiac valve papillary fibroelastoma: Surgical excision for revealed or potential embolization. Journal of Thoracic and Cardiovascular Surgery. 1999;117(1):106-10.

7. Ngaage DL, Mullany CJ, Daly RC, Dearani JA, Edwards WD, Tazelaar HD, et al. Surgical Treatment of Cardiac Papillary Fibroelastoma: A Single Center Experience With Eighty-Eight Patients. Annals of Thoracic Surgery. 2005;80 (5):1712-8.

8. Yee HC, Nwosu JE, Lii AD, Velasco M, Millman A. Echocardographic Features of Papillary Fibroelastoma and Their Consequences and Management. American Journal of Cardiology 1997;80(6):811-4. 